\title{
Novel MMC microstructure with tailored distribution of the reinforcing phase
}

\author{
H. X. PENG*, Z. FAN* \& J. R. G. EVANS $\dagger$ \\ *Department of Materials Engineering, Brunel University, Uxbridge, Middlesex UB8 3PH, U.K. \\ $\dagger$ Department of Materials, Queen Mary and Westfield College, University of London, \\ Mile End Road, London E1 4NS, U.K.
}

Key words. Aluminium matrix composite, damage tolerance, distribution, energy absorption, fibre agglomerate.

\section{Summary}

Saffil short fibre agglomerates with diameters of $0.4 \mathrm{~mm}$ to $1 \mathrm{~mm}$ have been prepared using a tumbling technique. These were packed and infiltrated with molten $6061 \mathrm{Al}$ alloy to make a metal matrix composite (MMC) with a novel microstructure in which the composite spheres are randomly distributed in the fibre-free aluminium matrix. In parallel, a commercial preform made of identical Saffil alumina short fibres and having the same fibre volume faction was used to prepare a conventional MMC by the same technique. Microstructural observation indicates that, within the composite spheres, the local volume fraction of fibre decreases from the outer layer to the centre region.

The energy absorption during fracture was estimated by using a three point loading test on notched samples and was compared with that for conventional MMCs. Preliminary results suggest that this novel MMC possesses higher energy absorption capability and hence better damage tolerance. The fracture surfaces were examined by scanning electron microscopy in order to inform these experimental results.

\section{Introduction}

The considerable interest in discontinuously reinforced metal matrix composites (MMCs) is the result of a fine balance of factors such as cost, damage tolerance (toughness, ductility, and flaw sensitivity), isotropy, thermal characteristics, reproducibility, environmental resistance, forming, machining and joining requirements. The attractive physical and mechanical properties that can be obtained with such MMCs have been documented and reviewed extensively (Kelly, 1985; Ibrahim et al., 1991; Lloyd, 1994; Sinclair \& Gregson, 1997). Despite the many

Correspondence to: Professor J. R. G. Evans. Fax: +44 (0)20 8981 9804;

e-mail: j.r.g.evans@qmw.ac.uk favourable attributes, these MMCs exhibit low room temperature damage tolerance. Their more widespread application has not been limited by basic load-bearing characteristics but by the dramatic reduction in damage tolerance that occurs on adding appreciable volume fractions of discontinuous brittle ceramic to metallic alloys (Van Stone et al., 1985).

In recent years, there has been interest in the unique design opportunities afforded by the controlled distribution of reinforcements at an intermediate or mesoscopic scale, specifically in terms of graded and layered structures (Nardone et al., 1991a, b). The laminate approach has shown potential as a toughening mechanism for particulatereinforced aluminium composite materials (Ellis \& Lewandowski, 1994; Mclelland et al., 1999). However, the measured toughness of the laminate is known to be affected by test orientation, i.e. the material is anisotropic just like continuously reinforced MMCs.

The damage tolerance of MMCs is important if these materials are to be used as structural components where improved structural reliability is required. The basic measure used to assess structural reliability in the current study is the energy absorbing capability of the composite during notched Charpy impact testing. The limited work that has been published on impact behaviour identifies a large loss of energy-absorbing capabilities accompanying reinforcement addition (Nardone et al., 1991b; Surappa \& Sivakumar, 1993). The brittle reinforcement generally decreases both the initiation and growth fracture toughness as measured by conventional tests (Flom \& Arsenault, 1989; Kamat et al., 1989; Manoharan. \& Lewandowski, 1990). Regarding the effects of structural variables on the fracture of such MMCs, attention has focused on altering volume fraction, size and aspect ratio of the reinforcing phase while processing to give a homogeneous distribution. However, theoretical analysis (Fan et al., 1992; Fan \& Miodownik, 1993) and published experimental results (Sinclair \& Gregson, 1997; Peng et al., 
2000; Zhou et al., 2000) indicate that local reinforcement distribution patterns can play a very significant role in controlling MMC properties as well as damage tolerance.

In the case of layered structures, crack growth resistance in orientated inhomogeneous microstructures can be developed and controlled via extrinsic crack shielding phenomena. By tailoring the distribution of the reinforcement, the reinforced/unreinforced interface can be specifically weakened to promote crack deflection (Ellis \& Lewandowski, 1994), but the intrinsically anisotropic behaviour and the dependence of shielding efficiency on precise loading geometry should be recognized. There are obvious advantages in fabricating such materials with an isotropic microstructure.

Saffil alumina short fibre is one of the most popular reinforcements for MMC production, and the composites produced therefrom have been used, inter alia, in automobiles and sports goods. The large safety margins required in the aerospace industry are difficult to satisfy and currently no discontinuous MMC components are flying in commercial aircraft. Furthermore, in traditionally squeezecast composites, the fibre distribution is still not isotropic because of the preform preparation process in which the fibres tend to align in the plane normal to the sedimentation and pressing direction (Clyne \& Withers, 1993).

This paper presents some of our work on the processing of MMCs with tailored microstructures provided by the fibre arrangement of the ceramic preform. It describes a novel processing technique that gives a distinct and original microstructure. The structure of the preform and of the MMC that results from its infiltration are described and finally a preliminary evaluation of the damage tolerance characterized by the energy absorption capability is presented.

\section{Experimental details}

\subsection{Starting materials}

The ceramic fibre selected for this study was Saffil alumina short fibre, which is predominantly $\delta$-alumina $\left(\mathrm{Al}_{2} \mathrm{O}_{3}\right)$ with an average diameter of $3 \mu \mathrm{m}$ and a fibre length ranging from $100 \mu \mathrm{m}$ to $150 \mu \mathrm{m}$. This was kindly donated by Vernaware, Bolton, U.K. Table 1 gives the basic properties of the fibre and its morphology is shown in Fig. 1. The matrix material used was 6061 aluminium alloy with $(0.8-$ $1.2) \% \mathrm{Mg}(0.4-0.6) \% \mathrm{Si}$ supplied by EMS (Uxbridge, Middlesex, U.K.). This alloy has liquidus and solidus temperatures of $650{ }^{\circ} \mathrm{C}$ and $582{ }^{\circ} \mathrm{C}$, respectively.

\subsection{Fibre-ball preparation and MMC fabrication}

The fibre agglomerates were manufactured by tumbling the as-received short fibres in a polyethylene container without
Table 1. Properties of Saffil alumina fibre (RF grade).

$\begin{array}{ll}\text { Chemical composition } & \mathrm{Al}_{2} \mathrm{O}_{3}: 96-97 \% \\ & \mathrm{SiO}_{2}: 3-4 \% \\ \text { Crystal phase } & \mathrm{Mainly} \delta \text {-alumina* }^{*} 3300 \mathrm{~kg} \mathrm{~m}^{-3} \\ \text { Density } & 3 \mu \mathrm{m} \\ \text { Median fibre diameter } & >2000{ }^{\circ} \mathrm{C} \\ \text { Melting point } & 2000 \mathrm{MPa} \\ \text { Tensile strength } & 300 \mathrm{GPa} \\ \text { Young's modulus } & \end{array}$

* Alpha alumina $<15 \%$, as claimed by the manufacturer.

grinding media at $80 \mathrm{rev} \mathrm{min}^{-1}$ for $10 \mathrm{~h}$. A narrow size distribution of agglomerates $(0.4-1 \mathrm{~mm})$ was selected by sieving. These were packed in a steel die to make a preform with a volume fraction of $10 \mathrm{vol} . \%$ and then infiltrated by liquid $6061 \mathrm{Al}$ alloy using a modified squeeze-caster with a fine infiltration speed, pressure control and fitted with a vacuum system (Dassett Engineering Corporation, U.K.). After the infiltration process, a maximum pressure of $65 \mathrm{MPa}$ was maintained until the die cooled and the aluminium phase was solidified. For comparison, the conventional MMC with the same fibre volume fraction was also prepared using the as-received preform (also supplied by Vernaware). This is made of the same short fibres.

\subsection{Microstructural characterization and damage tolerance evaluation}

The morphology of the fibre agglomerates and the microstructures of the composites were examined using a JEOL JXA840 SEM.

Test specimens with dimensions of $10 \times 8 \times 55 \mathrm{~mm}$ were cut from the as-cast billets. ' $V$ '-shaped notches were machined on the $10 \times 55 \mathrm{~mm}$ side to a depth of $2 \mathrm{~mm}$. At

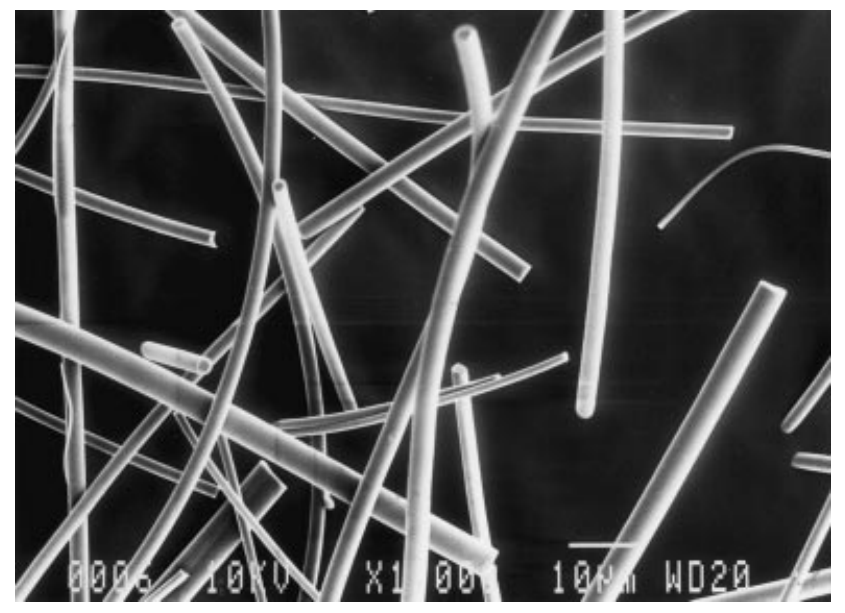

Fig. 1. Morphology of Saffil alumina short staple fibres. 


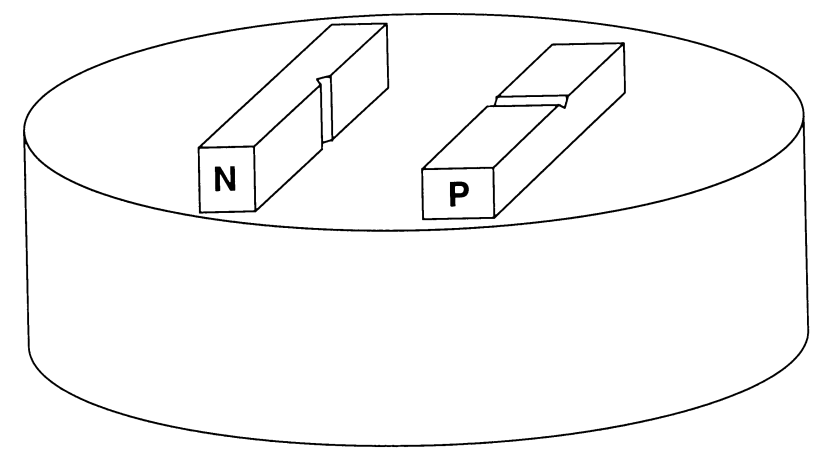

Fig. 2. Schematic configuration of the notched samples in relation to the cast billet.

10 vol.\% alumina, tool wear is not severe; nevertheless cuts were sequenced on samples from different materials to spread its effect. For the conventional MMC, due to its intrinsically anisotropic structure, the notches were machined in two ways, as shown in Fig. 2.

Three-point bending tests were performed on these samples at a span of $40 \mathrm{~mm}$ and constant cross-head speed of $0.1 \mathrm{~mm} \mathrm{~min}{ }^{-1}$ using an Instron Model 4206 testing machine fitted with a $5 \mathrm{kN}$ load cell and a three-point loading fixture of $10 \mathrm{kN}$ capacity designed by Instron. The load-displacement curves were recorded by an on-line computer system. The experiment was terminated when the load was below $20 \mathrm{~N}$. The fracture surfaces were observed by SEM.

\section{Results and discussion}

\subsection{Microstructural characterization of the composites}

The morphology of the fibre agglomerates is shown in Fig. 3(a) and the fibre arrangement on the surface of the ball is shown in Fig. 3(b). The fibre agglomerates are generally intact, unbroken and uniformly spherical. It can be seen that, within the fibre assembly, the fibres are randomly distributed. After classification, the diameters of the fibre agglomerates ranged from 0.4 to $1 \mathrm{~mm}$ which is relatively fine when compared with the length of the fibres $(<200 \mu \mathrm{m})$.

Figure 4(a) shows the microstructure of the composite prepared from the fibre agglomerates, while the microstructure of the conventional MMC prepared from the as-received fibre preform is shown in Fig. 4(b). Under the assistance of the die vacuum system, the fibre agglomerates were thoroughly infiltrated by molten aluminium to form composite spheres. It can be seen that these composite spheres are uniformly distributed in the aluminium matrix at the macroscopic level. The material has an isotropic microstructure. By contrast, the conventional MMC reveals a planar random fibre distribution throughout the aluminium matrix, as shown in Fig. 4(b).
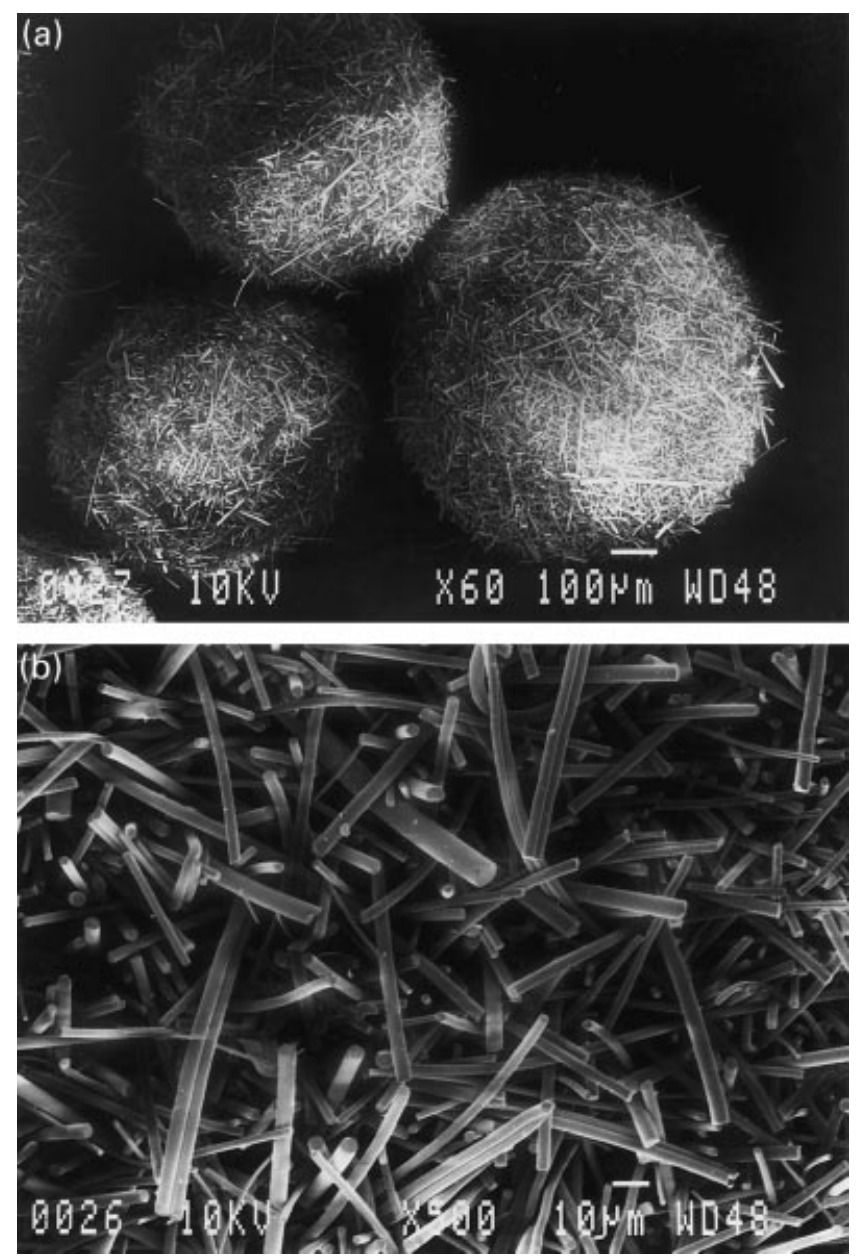

Fig. 3. (a) The morphology of the fibre agglomerates and (b) the fibre arrangement on the surface.

Further examination of the microstructure of this newly developed MMC (Fig. 5) indicated that the fibre distribution within the composite sphere varies from the edge to the centre along the diameter. A higher volume fraction of fibres is observed in the outer layers of the sphere, whereas the local volume fraction in the centre is lower. In order to quantify this, a number of spheres that had the largest sections and therefore had the highest probability of presenting a diameter on the polished surfaces were analysed along their apparent diameters from one side to the other by using a Q520 Image Analyser (Cambridge Instruments, Cambridge, U.K.). The local volume fraction was plotted as a function of the relative position on a normalized sphere diameter, as shown in Fig. 6. This indicates that the local fibre volume fraction in the outer region can be as high as $30 \%$, whereas the fibre volume fraction in the central region is about $15 \%$. This interesting distribution provides a more rigid outer layer in the composite spheres due to the higher volume fraction compared with that in the central region. In addition, the volume fraction of the composite 

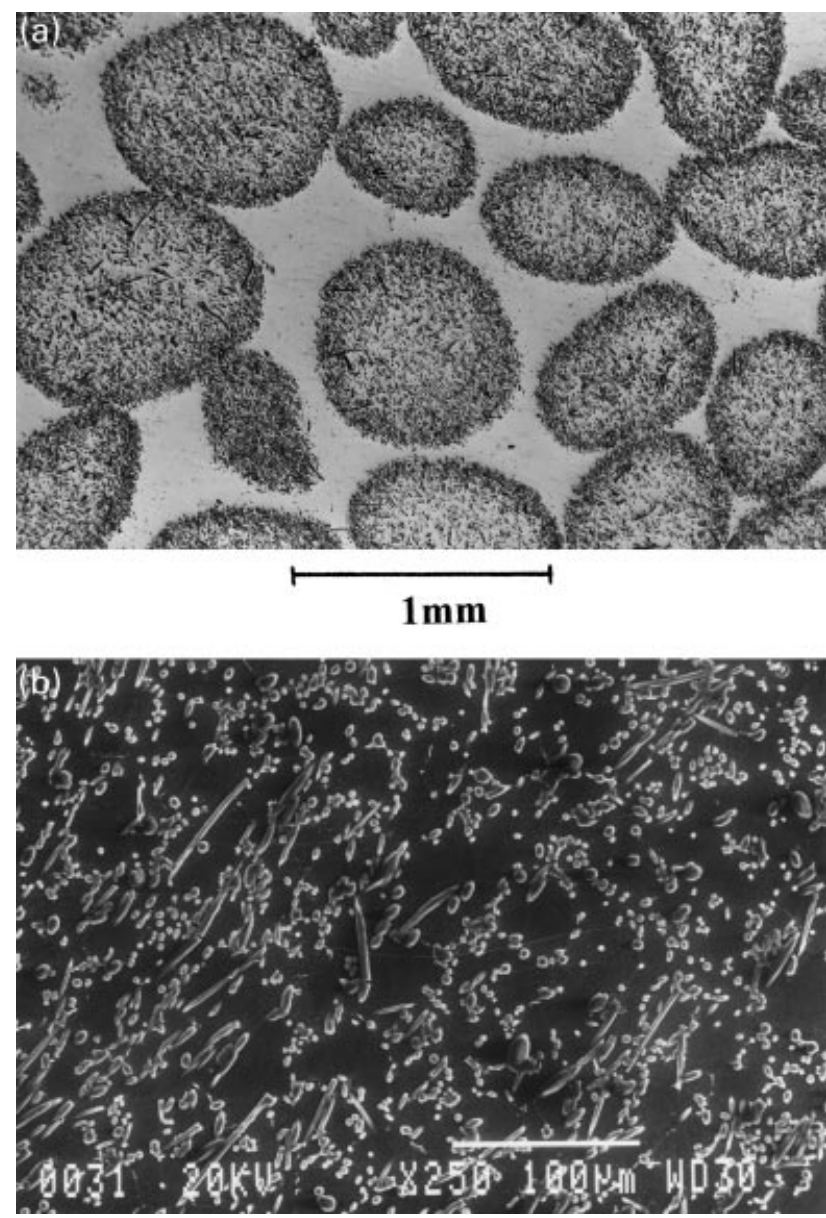

Fig. 4. (a) The microstructure of the composite prepared from fibre agglomerates and (b) the microstructure of a conventional MMC prepared from the as-received fibre preform.

spheres is approximately $64 \%$ based on the whole composite and was obtained by measuring the area fraction of spheres.

From Fig. 5, it is also important to note that the interface between the composite spheres and the unreinforced aluminium is not a sharp interface such as that which exists in conventional MMCs between two dissimilar materials. There is a transition from the fibre-free region to the composite sphere. It is evident that any sharp interface between dissimilar materials can act as an effective stress concentrator under mechanical or thermal loading, limiting the performance potential of the overall system. The gradation of the interface between dissimilar materials may thus be advantageous.

\subsection{Three-point loading test and the damage tolerance of the composites}

Figure 7 shows the typical load-displacement curve of the conventional MMC and the newly developed MMC obtained during the flexural tests in which the conventional MMC

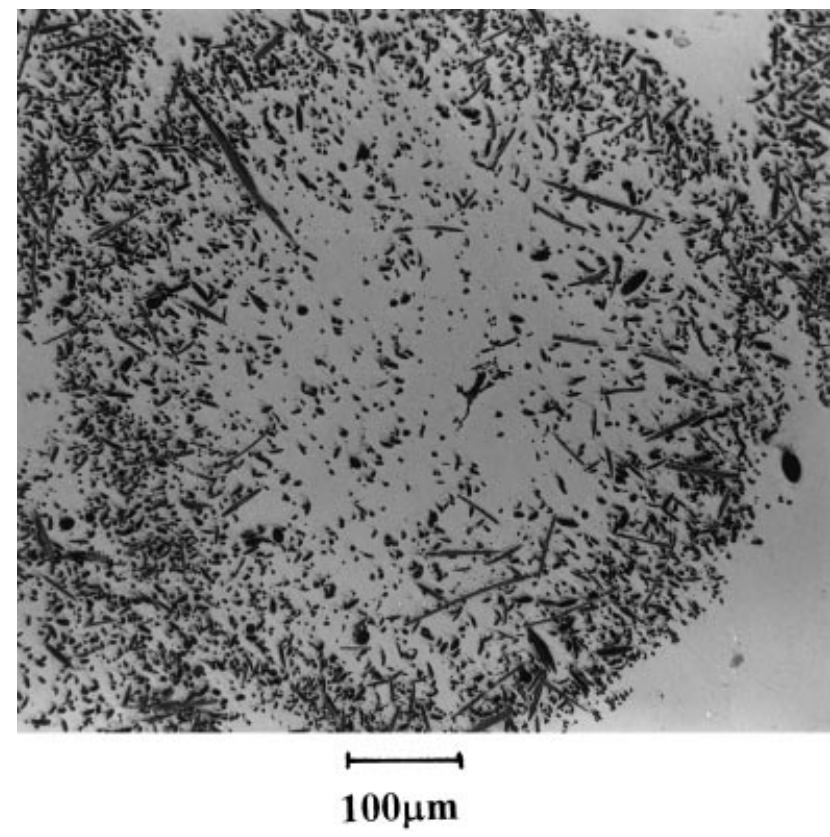

Fig. 5. Scanning electron micrograph of a composite sphere showing the variation in the fibre volume fraction.

was tested in the two directions denoted $\mathrm{N}$ and $\mathrm{P}$ as defined in Fig. 2. The total area below the curve represents the energy absorbed during the bending process. As expected, the sample denoted $\mathrm{P}$, in which the notch was parallel to the random fibre plane, sustained a higher maximum load than that denoted $\mathrm{N}$, in which the notch was normal to the random fibre plane. Also sample $\mathrm{P}$ absorbed more energy than sample $\mathrm{N}$. The novel MMC developed in this work sustained an equivalent maximum load to sample $\mathrm{N}$; the value is slightly less than that obtained from sample P.

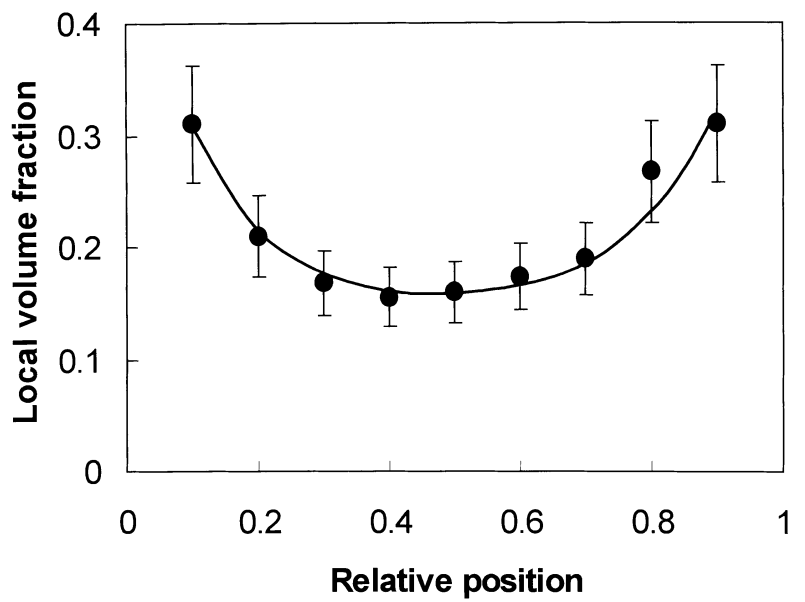

Fig. 6. Variation in local volume fraction of fibres within the composite spheres with respect to the relative position on the sphere diameter (each point is the mean of at least six measurements: bars represent 95\% confidence limits). 


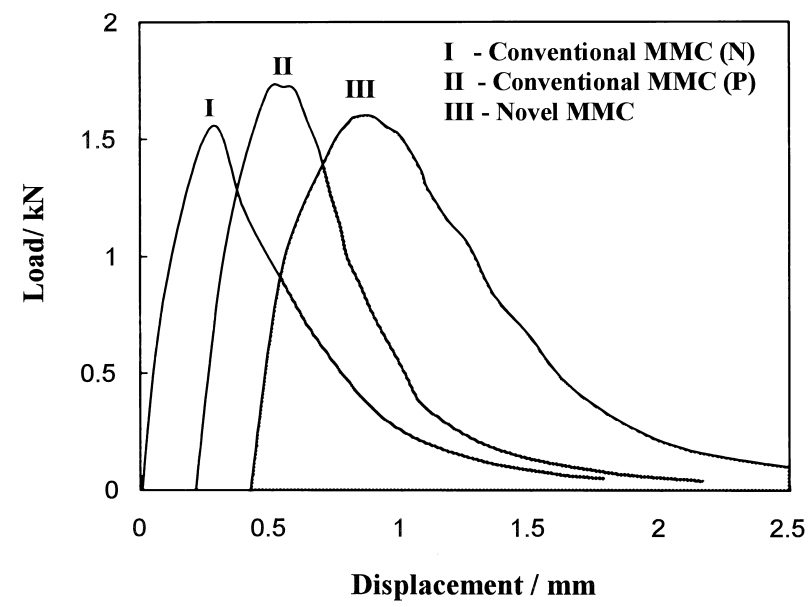

Fig. 7. Typical load-displacement curve of the conventional MMC and of the novel MMC.

Table 2. The energy absorbed by the composite during bending test.

Materials

Total energy to break, $G_{b}(J)$

\begin{tabular}{ll} 
Conventional-N & 0.90 \\
Conventional-P & 1.19 \\
Novel MMC & 1.53 \\
\hline
\end{tabular}

However, this material gave the largest area below the curve as shown in Fig. 7, indicating that more energy was absorbed during identical flexural tests. Interestingly, the additional energy absorbed by the novel composite was recorded after the peak load. The total energy absorbed is shown in Table 2 and is about 50\% higher than that for the conventional MMC.
In order to inform these measurements, the crack propagation route of the novel MMC was examined by SEM and is shown in Fig. 8. The crack tends to propagate along the interfaces between aluminium and the composite spheres providing significant crack deflection. This resulted mainly from the reinforced outer layer of the composite sphere, which can apparently carry enough load to discourage the crack from going directly through the sphere until a higher stress is generated. Clearly, when the crack deflection is far away from the main crack direction, the crack passes through the composite spheres, as observed in Fig. 8. As shown in Fig. 5, within the composite sphere, the local fibre volume fraction is lower, and this provides higher crack propagation resistance and hence good damage tolerance.

The observations of the fracture surfaces of the novel MMC are shown in Fig. 9. The fractograph in Fig. 9(a) is mirrored by an identical image taken from the countersurface (Fig. 9b). The fracture surfaces are very rugged. It seems that the fracture occurred near the interfaces between the unreinforced aluminium and composite sphere and resulted in either a protruding composite ball or a concave socket yielding a tortuous crack propagation path. Compared with the fracture surfaces of conventional MMCs these fractograms suggest substantial crack deflection that may contribute to the enhanced energy absorption. Further investigations are ongoing to explore the strength and ductility of this and other novel MMC structures.

\section{Conclusions}

By employing Saffil alumina short fibres, fine alumina fibre agglomerates have been manufactured by a tumbling technique. These fibre agglomerates were then employed to make a novel MMC, which has a hierarchy microstructure

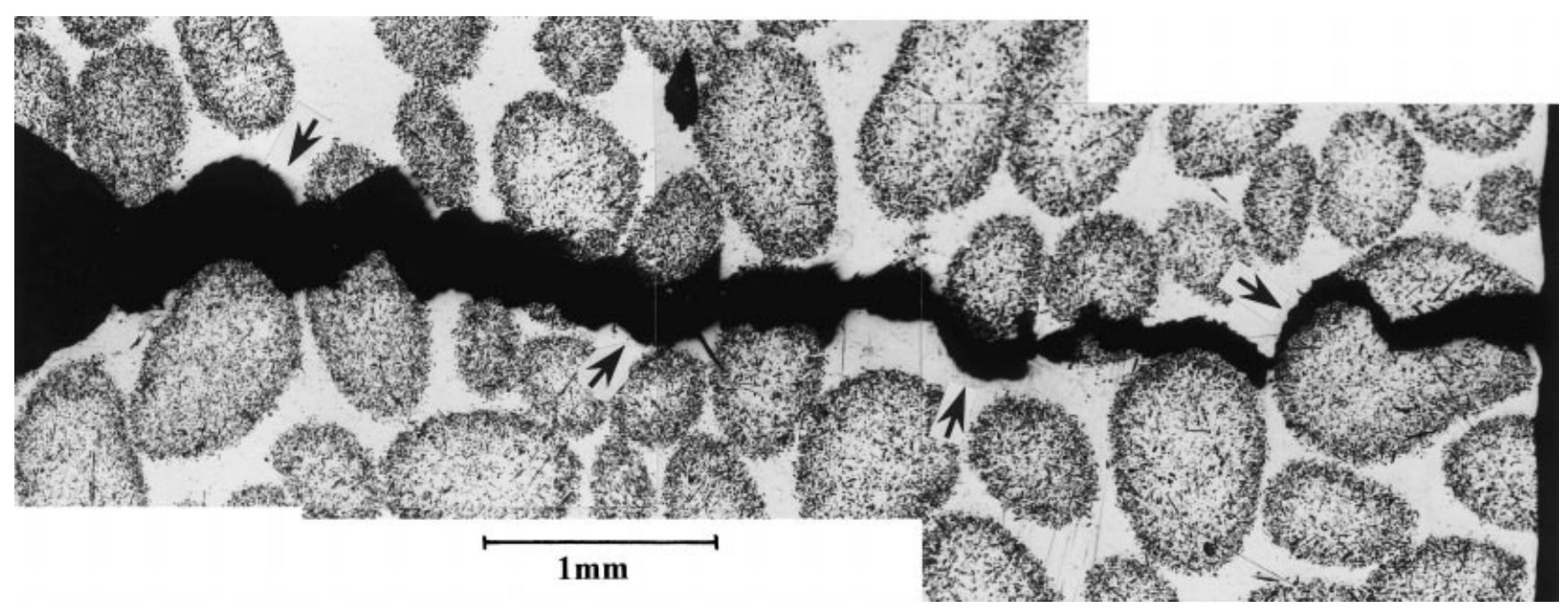

Fig. 8. The crack propagation route of the novel MMC during fracture. 

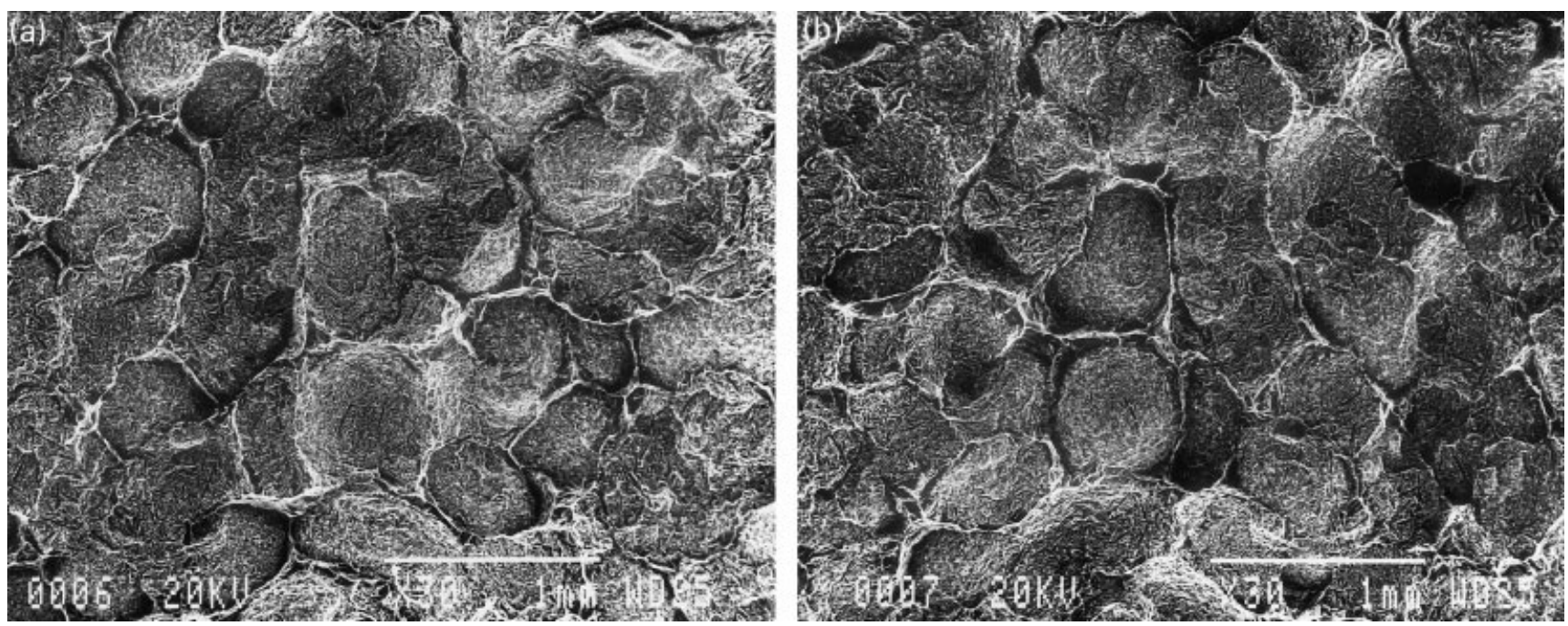

Fig. 9. Matching fracture surfaces of the novel MMC showing the composite sphere pull out.

in which MMC spheres are uniformly distributed in a fibrefree $\mathrm{Al}$ matrix in contrast to the conventional MMC that has a uniform planar random distribution of short fibres.

Microstructural observation indicates that, within the composite spheres, the local volume fraction of fibre $\left(V_{f}\right)$ exhibits a gradient pattern with a higher $V_{f}$ in the outer layer region and a lower $V_{f}$ in the central region.

Three-point loading tests on notched samples can be used to assess the energy absorption capability of the composites. Compared with conventional MMCs, the newly developed MMC possesses the superior energy absorption capability and hence a better damage tolerance. This is mainly due to the tailored reinforcement distribution, which can improve the crack propagation resistance mainly through crack deflection.

\section{Acknowledgements}

The authors are grateful to EPSRC for the financial support of this work under Grant No. GR/L80553.

\section{References}

Clyne, T.W. \& Withers, P.J. (1993) An Introduction to Metal Matrix Composites. 2nd edn. Cambridge University Press, Cambridge.

Ellis, L.Y. \& Lewandowski, J.J. (1994) Effects of layer thickness on impact toughness of Al/Al-SiCp laminates. Mater. Sci. Eng. A183, $59-67$.

Fan, Z. \& Miodownik, A.P. (1993) The deformation behaviour of alloys comprising two ductile phases. Acta Metall. Mater. 41, 2403-2413, 2415-2423.

Fan, Z., Tsakiropoulos, P. \& Miodownik, A.P. (1992) Predication of Young's modulus of particulate two phase composites. Mater. Sci. Technol. 8, 922-929.

Flom, Y. \& Arsenault, R. (1989) Effect of particle size on fracture toughness of $\mathrm{SiC} / \mathrm{Al}$ composite material. Acta Metall. 37, 24132423.
Ibrahim, I.A., Mohamed, F.A. \& Lavernia, E.J. (1991) Particulate reinforced metal matrix composites - a review. J. Mater. Sci. 26, 1137-1156.

Kamat, S.V., Hirth, J.P. \& Mehrebian, R. (1989) Mechanical properties of particulate-reinforced aluminium matrix composites. Acta Metall. 37, 2395-2402.

Kelly, A. (1985) Composites in context. Compos. Sci. Technol. 23, 171-199.

Lloyd, D.J. (1994) Particle reinforced aluminium and magnesium matrix composites. Int. Mater. Reviews, 39, 1-24.

Manoharan, M. \& Lewandowski, J.J. (1990) Crack initiation and growth toughness of an aluminium metal-matrix composite. Acta Metall. Mater. 38, 489-496.

Mclelland, A.R.A., Atkinson, H.V. \& Anderson, P.R.G. (1999) Thixoforming of a novel layered metal matrix composite. Mater. Sci. Technol. 15, 939-945.

Nardone, V.C., Strife, J.R. \& Prewo, K.M. (1991a) Processing of particulate reinforced metals and intermetallics for improved damage tolerance. Mater. Sci. Eng. A144, 267-275.

Nardone, V.C., Strife, J.R. \& Prewo, K.M. (1991b) Microstructurally toughened particulate-reinforced aluminium matrix composites. Metall. Trans. 22A, 171-182.

Peng, H.X., Fan, Z. \& Evans, J.R.G. (2000) Novel MMC microstructures prepared by melt infiltration of reticulated ceramic preforms. Mater. Sci. Technol. 16, 903-907.

Sinclair, I. \& Gregson, P.J. (1997) Structural performance of discontinuous metal matrix composites. Mater. Sci. Techol. 13, $709-726$.

Surappa, M.K. \& Sivakumar, P. (1993) Fracture-toughness evaluation of $2040-\mathrm{Al} / \mathrm{Al}_{2} \mathrm{O}_{3}$ particulate composites by instrumented impact. Compos. Sci. Technol. 46, 287-292.

Van Stone, R.H., Cox, T.B., Low, J.R. \& Psioda, J.S. (1985) Microstructural aspects of fracture by dimpled rupture. Int. Mater. Rev. 30, 157-179.

Zhou, Z., Peng, H.X., Fan, Z. \& Li, D.X. (2000) MMCs with a controlled non-uniform distribution of submicron $\mathrm{Al}_{2} \mathrm{O}_{3}$ particles in a 6061Al matrix. Mater. Sci. Technol. 16, 908-912. 